\title{
Photoelectron Circular Dichroism at the Few-cycle Limit
}

\author{
Václav Hanus ${ }^{1, *}$, Sarayoo Kangaparambil ${ }^{1}$, Seyedreza Larimian $^{1}$, Maurice Tia $^{1}$, Xinhua \\ $\mathrm{Xie}^{1}$, Sebastian Eckart ${ }^{2}$, Markus Schöffler ${ }^{2}$, Andrius Baltuska ${ }^{1}$ and Markus Kitzler ${ }^{1}$ \\ ${ }^{1}$ Photonics Institute, Technische Universität Wien, Gusshausstrasse 27, 1040 Wien, Austria \\ ${ }^{2}$ Institut für Kernphysik, J.W. Goethe-Universität, Max-von-Laue-Strasse 1, 60438 Frankfurt \\ am Main, Germany
}

\begin{abstract}
Photoelectron circular dichroism (PECD) was investigated for methyloxirane with intense few-cycle pulses. The observed PECD shows a dependence on electron energy. This may signify an influence of the chiral potential on the outgoing electron's trajectory.
\end{abstract}

\section{Introduction}

Chirality is a property of matter that discriminates objects into two types: left-handed and right-handed which are mirror counterparts of each other. The identification of a specific handedness requires processes that are sensitive to this property of matter and offer so called chiral recognition. One of such processes that features this chiral recognition is photoelectron circular dichroism (PECD). This process leads to a forward-backward asymmetry (with respect to the laser propagation direction) in photoelectron distributions created upon interaction of a circularly or elliptically polarized laser with a chiral object. It was shown that the laser induced PECD is a universal effect that is independent of the ionization regime. It can be observed in all ionization regimes, from single- over multiphoton ionization until the tunnel ionization regime, and has been shown to be sensitive to dynamical changes of the structure of chiral molecules [1]. The fact that the effects of chirality can be observed in the tunnel ionization regime opens up the possibility to connect this fundamental effect to strong-field science with prospects for studying and driving sub-laser-cycle and attosecond phenomena connected to PECD. However, a thorough understanding of PECD in the tunneling regime is of yet missing. It was speculated that it emerges because of multiple scattering of the electron trajectory off the chiral molecular potential during the ionization process [1].

Here, we pick up on this point and investigate PECD using few-cycle laser pulses with the aim of obtaining signatures of the influence of electron trajectories in asymmetries of photoelectron momentum distributions. We find a dependence of the characteristic photoelectron-asymmetry on electron energy, which may be interpreted as a signature of the influence of the outgoing electron's trajectory on PECD.

\footnotetext{
* Corresponding author: vaclav.hanus@tuwien.ac.at
} 


\section{Experiment and Results}

PECD is a rather weak effect. Thus, it is favorable to perform PECD measurements with techniques that are able to improve the chiral recognition. One of them is coincidence detection of ions and electrons [2], which opens up the possibility to further enhance the strength of PECD by orientation of fragmenting molecules during data analysis [3]. Here we use coincidence detection using the COLTRIMS technique. We measure the momentum of electrons emitted during the interaction of near-circularly polarized laser pulses with a duration of $\sim 5 \mathrm{fs}$ with the chiral molecule methyloxirane (also known as 1,2-propylene oxide). The generated ions were detected in coincidence with the electrons. Low laser intensity $\left(1 \times 10^{14} \mathrm{~W} / \mathrm{cm}^{2}\right)$ was used to obtain dominantly singly ionized molecules. Measurements were done with enantiopure samples (which contained only one enantiomer of the molecule, denoted $\mathrm{S}$ and $\mathrm{R}$ ) and near-circular laser polarization states with opposite helicities, denoted as A and B, respectively. Switching between helicities was performed by rotating an achromatic quarter wave-plate.
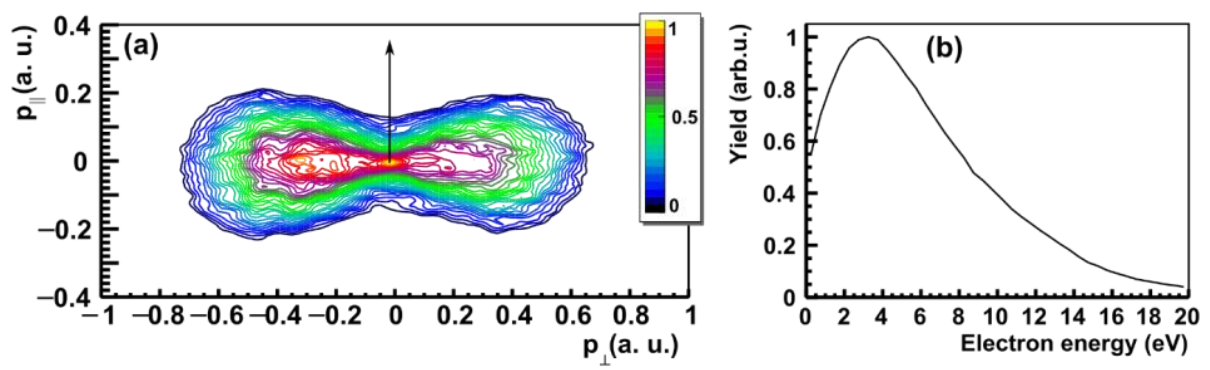

Fig. 1. (a) Yield of electrons measured in coincidence with singly ionizaed methyloxirane in the plane defined by the laser propagation direction (vertical axis) and the direction of minor axis of polarization ellipse (horizontal axis). (b) Energy distribution of electrons.

Fig. 1(a) shows the momentum distribution of photoelectrons for sample $\mathrm{S}$ and helicity $\mathrm{B}$ in a plane perpendicular to the laser polarization plane, spanned by the electron momentum component parallel to the laser propagation direction, $p_{\|}$, and the momentum component along the minor axis of the laser polarization ellipse, $p_{\Perp}$. The PECD parameter for a certain polarization state (A or B) is calculated as the normalized difference of the yield of electrons between the two enantiopure samples, denoted as $N_{S}$ and $N_{R}$. The asymmetry of this parameter due to the PECD effect is calculated with respect to the angle $\theta$ defined as the angle between the laser propagation direction and the momentum of a given electron, $\theta=\operatorname{atan}\left(\frac{p_{\Perp}}{p_{\mathbb{I}}}\right)$,

$$
P E C D_{A}, P E C D_{B}=\frac{\mathbb{N}_{S}(\theta)-\mathbb{N}_{R}(\theta)}{\mathbb{N}_{S}(\theta)+\mathbb{N}_{R}(\theta)} .
$$

The narrow shape of the electron momentum distribution in Fig. 1(a) is very similar to that observed in the tunneling regime (although the Keldysh parameter indicates that we are not in tunnel regime here). In the following we will discuss the dependence of the asymmetry of the PECD parameter with respect to $\theta$ on the electron energy.

\section{Dependence of PECD on photoelectron energy}

The electron momentum distribution resolved in the angle $\theta$ of the momentum and the laser propagation direction shows a clear asymmetry between two chiral samples. This is visible 
in Fig. 2(a), which shows the asymmetry parameter from Eq. 1 integrated over all electron energies. The slope of the line shows the strength of the PECD. The slope is positive for PECD measured with helicity A and negative with helicity $\mathrm{B}$. The momentum distribution of electrons [see Fig. 1(a)] is elongated along $90^{\circ}$, parallel to the plane of the laser polarization. The yield shows a minimum along $\theta=0^{\circ}$ and $\theta=180^{\circ}$. Therefore, in order to avoid high statistical errors, the range of the angle in Fig. 2 is limited to $50^{\circ}$ to $130^{\circ}$. In Fig. 2(b) we plot the PECD parameter for certain energy ranges of the photoelectrons. From this figure it becomes clear that for low photoelectron energy one observes smaller asymmetry than the integral one. Although only the case of B helicity is shown, we observe the same behavior in measurements with helicity A.
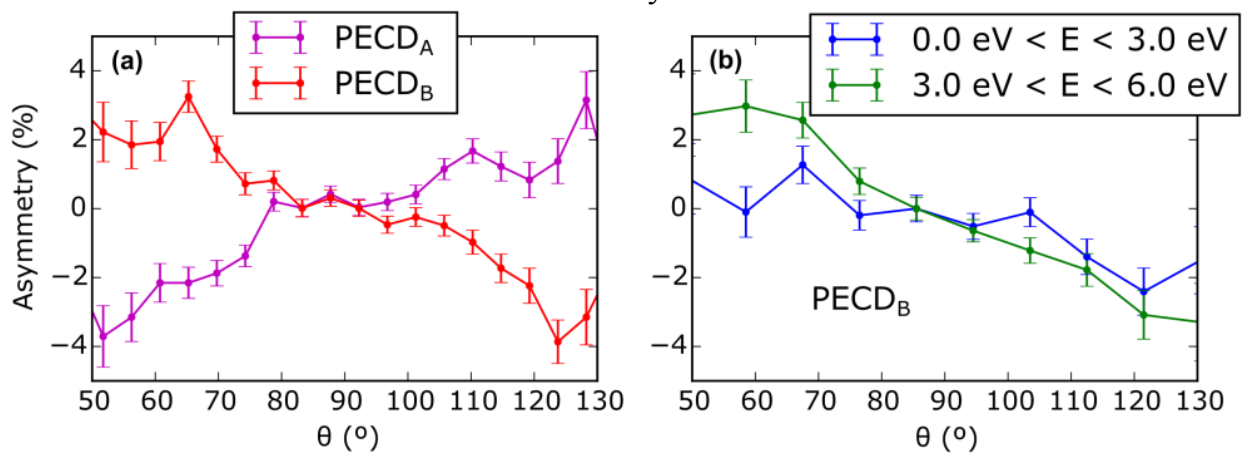

Fig. 2. (a) Measured PECD asymmetry as defined in Eq. (1) as a ratio of the yield of electrons for sample $\mathrm{S}$ and R. $\theta$ is the angle between the momentum of the electron and the laser propagation direction. PECD asymmetry inverts sign when helicity of laser is changed. (b) PECD for the case of helicity B plotted for two different photoelectron energy ranges.

\section{Discussion and Outlook}

We measured PECD of a chiral molecule with an intense few-cycle laser pulse employing coincidence detection of photoelectrons and -ions. We found a dependence of the PECD parameter on the electron energy. Because in strong laser fields the electron energy constitutes a measure of the emission time of the electron and its trajectory to the detector, this result may be interpreted as a signature of the influence of the chiral molecular potential on the electron's trajectory. Because the electron's trajectory also depends on the sub-cycle evolution of the laser field, this interpretation may be scrutinized by studying the influence of the few-cycle laser pulses' carrier envelope phase (CEP) on the PECD parameter and its energy dependence. Further prospects would be dynamical control of the PECD using the CEP.

\section{References}

1. S. Beaulieu et al., "Universality of photoelectron circular dichroism in the photoionization of chiral molecules," New J. Phys. 18, 102002 (2016).

2. M. Pitzer et al., "Direct Determination of Absolute Molecular Stereochemistry in Gas Phase by Coulomb Explosion Imaging," Science 341, 1096-1100 (2013).

3. M. Tia et al., "Observation of Enhanced Chiral Asymmetries in the Inner-Shell Photoionization of Uniaxially Oriented Methyloxirane Enantiomers," J. Phys. Chem. Lett. 8, 2780-2786 (2017). 